THE METABOLISM OF MARINE ANIMALS. Die Ernährung der Wassertiere und der Stoffhaushalt der Gewasser. By Prof. August Pütter. Pp. iv + 168. (Jena: Gustav Fischer, 1909.) Price 5 marks (unbound).

TWO years ago Prof. Pütter published three papers dealing with the metabolism of marine animals. The thesis advanced as the result of these investigations may be briefly summarised as follows:- the nutrition of a very great number of marine animals belonging to all phyla is not effected in the manner characteristic of the mammal, that is, by the ingestion of solid organised food, and by the subsequent digestion and absorption of this matter by special organs, but by the direct absorption of carbon and nitrogen compounds which are contained in solution in the sea. The notion that many animals were really saprozoic in their habits was not really a new one; most internal parasites, whether provided or not with an alimentary canal, obviously exhibit such a mode of nutrition; but the hypothesis that animals living in the open feed otherwise than by the ingestion of solid organised food, or by the utilisation of photosynthetic products elaborated by the activity of commensal algæ, was a new one, and has provoked much discussion. Pütter's methods have been criticised by Henze and Lohmann, and the paper now under review amplifies the author's former work, and to some extent meets the criticisms advanced.

The proof of the thesis is developed along three main lines. The author has studied the intensity of metabolism in a number of forms, and has found that this is proportional to the unit of surface, and not to the unit of mass. Therefore the relatively minute organisms which are found among the plankton, or even those larger animals which are provided with a large absorptive surface in the shape of gills, ctenidia, respiratory plumes, \&c., and internal diverticula, are able to utilise the exceedingly dilute solution of organic carbon and nitrogen compounds contained in sea water. The intensity of metabolism is measured by the oxygen consumption and the carbonic acid output, and, generally speaking, the rate of exchange is, roughly, constant in animals of the same general type of organisation, when it is regarded as a function of the unit of surface. The divergencies from this approximately constant rate are to be regarded as dependent on the deviations from the usual mode of metabolism characteristic of the animal group considered.

The second line of proof depends on the existence of compounds of carbon other than carbonates, and compounds of nitrogen other than ammonia, nitrates, and nitrites, in solution in sea water. From the author's point of view the sea is an immense storehouse of dissolved food-stuff, which is utilised by most marine animals. In his first papers, Pütter estimated that the water of Naples Bay contained some $6_{5}$ milligrams of organic carbon (volatile and higher fatty acids, and carbohydrates) per litre. Shortly afterwards Henze showed that the amount was greatly over-estimated, and that the proportion of such substances present was so small that it lay within the NO. 2 I 23 , VOL. 84$]$ limits of error of the experimental methods employed by Puitter. Raben, however, showed that the water from the North Sea and Baltic did actually contain measurable quantities of organic carbon varying from about 3 to 37 milligrams per litre. If these results should be confirmed, they would back up Pütter's hypothesis, since the solution would then be sufficiently concentrated to act as a food medium.

The third line of proof is much stronger, but it depends on the author's estimates of the rate of exchange of oxygen and carbonic acid in the animals studied. Taking the case of plankton-feeding creatures, he shows that it is, in most cases, impossible that a sufficient amount of food can be obtained from the plankton to account for the rate of metabolic exchange. A sponge (Suberites), for instance, of some 60 grams weight required about $0^{\circ} 9^{2} \mathrm{mgrm}$. of carbon per hour. Now taking a certain density of the plankton, this postulated that the sponge would have to pass some 242 litres of water through its canal system in order to get the necessary food-stuff from the plankton. It is quite impossible, of course, that the animal can filter this volume of fluid in the time. It has been shown by Lohmann that Pütter underestimated the density of the plankton, and by Henze that he over-estimated the concentration of the sea water in carbon compounds. But when the revised values are substituted, the argument is not materially affected. A further instance of the same nature is that of the copepod Calanus. If this animal feeds exclusively on plankton diatoms it must ingest some 16,000 medium-sized Coscinodisci, or about ten millions of Thalassiosiræ, in order to account for its metabolic exchange. Such figures appear to preclude the possibility of an exclusive feeding on diatoms.

It is, of course, quite probable that marine animals may feed in the same way as internal parasites; by absorption of dissolved food-stuff, and that this mode of nutrition may proceed simultaneously with that depending on the existence of an alimentary canal. If the metabolism of the lower invertebrates had been studied as carefully as that of the warm-blooded animal, this contention might have been accepted long ago. It is mainly by analogy with the latter that we ascribe respiratory functions to the structures called gills; they might just as reasonably be regarded as organs for absorption of food-stuff. However this may be, it appears from the work now noticed that the conclusions are only very probable ones until the data representing the rate of exchange of oxygen and carbonic acid have been critically revised. The proof or disproof of the author's thesis will be effected by such revision.

J. J.

\section{SMALLPOX AND VACCINATION IN BRITISH INDIA.}

Smallpox and Vaccination in British India. By Major S. P. James. Pp. xi+ro6. (Calcutta : Thacker, Spink and Co., 1909.) Price $7 s .6 d$. $A^{T}$ a time when the study of tropical diseases is A setting its indelible mark on the history of the progress of medicine, it is well to be reminded that 